\title{
A Lossless Data Compression System for a Real-Time Application in HEP Data Acquisition
}

\author{
Christian Patauner, Alessandro Marchioro, Sandro Bonacini, Attiq Ur Rehman, and Wolfgang Pribyl
}

\begin{abstract}
This paper presents a compression system optimized for the reduction of data from pulse digitizing electronics.

Such systems are widely used in High Energy Physics experiments for the collection of signals from calorimeters, Time Projection Chambers and in general all detectors that produce a signal amplified and/or shaped to a linear pulse. The Time Projection Chamber of the ALICE experiment will be used as an illustration of our method, which we believe is widely adaptable to other data acquisition chains in High Energy Physics.

To go beyond what can be achieved by conventional lossless compression schemes, which are ultimately limited by the intrinsic entropy of the incoming data, the proposed compression method makes use of a new scheme where instead of handling the data from the ADC as individual uncorrelated samples, an entire vector of samples is compressed.
\end{abstract}

Our method works by first approximating the incoming vectors formed by the digitization of the shaped pulses with a set of digitized reference vectors stored in a memory and retaining only the differences between these vectors using Huffman compression. In standard data compression language, this corresponds to a vector quantization combined with Huffman coding.

The performance of the described compression method was first evaluated by modeling the algorithm in Matlab using input data measured from the TPC in the ALICE experiment. A compression of $49 \%$ has been achieved. It has to be noted that the entropy computed on these same $10 \mathrm{bit}$ original data was only $6.2 \mathrm{bit} / \mathrm{symbol}$ thus allowing a maximum compression factor of $38 \%$ only.

The compression method was subsequently modeled in Verilog and synthesized to be combined with the other logic in the actual Read-out Control Unit of the ALICE TPC. First tests with actual hardware were performed using a Virtex-4 development board running at $80 \mathrm{MHz}$. The results showing the efficiency of the implementation using cosmic ray data are presented.

The algorithm is executed in this hardware with a latency of around 24 clock cycles at $40 \mathrm{MHz}$ and takes about 2000 logic slices to be implemented.

Index Terms-Data acquisition systems, data compression, front-end electronics of particle detectors, Huffman coding, vector quantization.

\section{INTRODUCTION}

D ATA compression is widely used in numerous computing and communication applications, ranging from text to music and video compression, but is relatively little used in

Manuscript received June 14, 2010; revised February 17, 2011; accepted March 31, 2011. Date of publication May 19, 2011; date of current version August 17, 2011.

C. Patauner is with the Institute of Electronics, Graz University of Technology, 8010 Graz, Austria, and also with the CERN-European Organization for Nuclear Research, 1211 Geneva 23, Switzerland (e-mail: christian.patauner@cern.ch).

A. Marchioro, S. Bonacini, and A. U. Rehman are with the CERN-European Organization for Nuclear Research, 1211 Geneva, Switzerland (e-mail: alessandro.marchioro@cern.ch; sandro.bonacini@cern.ch; attiq.ur.rehman@cern.ch).

W. Pribyl is with the Institute of Electronics, Graz University of Technology, 8010 Graz, Austria (e-mail: wolfgang.pribyl@tugraz.at).

Color versions of one or more of the figures in this paper are available online at http://ieeexplore.ieee.org.

Digital Object Identifier 10.1109/TNS.2011.2142193 data acquisition systems in particle physics. This possibly derives from two facts: first the very nature of data in particle physics often resembles random data even over a large number of samples, (and it is well known that random data can not very effectively be reduced) and second lossy schemes are often difficult to be implemented in real time. However, data acquisition systems in modern experiments could gain significantly from reducing data right at the source in terms both of bandwidth requirements and storage space. We have investigated one method of data compression using as a real application the data from the Time Projection Chamber (TPC) of the ALICE (A Large Ion Collider Experiment). Our method is well applicable also to other detector read-out systems, especially those where the front-end channels digitize pulses into several samples producing short vectors of data.

The ALICE TPC is the largest such detector built in recent years [1]. It consists of an $88 \mathrm{~m}^{3}$ cylinder filled with a gas mixture of $\left(\mathrm{Ne}-\mathrm{CO}_{2}-\mathrm{N}_{2}\right)$. The endplates are equipped with 560,000 small electrodes (size as small as $4 \mathrm{~mm} \times 7.5 \mathrm{~mm}$ ), which are connected to the front-end electronics. The electrodes measure the $x-y$ coordinates of the point of arrival of the electrons in the cylinder plane with a resolution of around $1 \mathrm{~mm}$ [2] and also the time of arrival of the electrons is sampled with a $10 \mathrm{MHz}$ clock [3]. The electronic chain presently equipping the read-out of this detector consists of a charge-sensitive shaping amplifier named PASA (PreAmplifier ShAper), a chip containing ADCs and digital processors called ALTRO (Alice Tpc Read Out) and a Read-out Control Unit (RCU). This chain is shown in Fig. 1. In case of ion beam collisions, around 20,000 particle tracks are expected to traverse the TPC for each bunch crossing. The Large Hadron Collider (LHC) will produce ion collisions with a frequency of $8 \mathrm{kHz}$. The rate of interesting events for the ALICE TPC is estimated at around $300 \mathrm{~Hz}$ and the event size is estimated at 66 Mbyte after the zero suppression [2], [3]. With these estimations, the maximum data rate for the TPC can be calculated as follows:

$$
D_{\text {rate }}=66 \text { Mbyte } \times 300 \mathrm{~Hz} \approx 20 \frac{\text { Gbyte }}{\mathrm{s}} .
$$

Considering that an experiment runs for years, this amount of data cannot be stored easily. Therefore, it is important to keep the amount of data as low as possible already in the front-end electronics as to reduce data transfer and storage requirements. The occupancy is expected to be low in ALICE and zero suppression is used for the first data reduction [4]. This mechanism reduces the amount of data significantly $(\approx 80 \%)$. Nevertheless, the data rate in (1) is estimated after the zero suppression and is still high. 


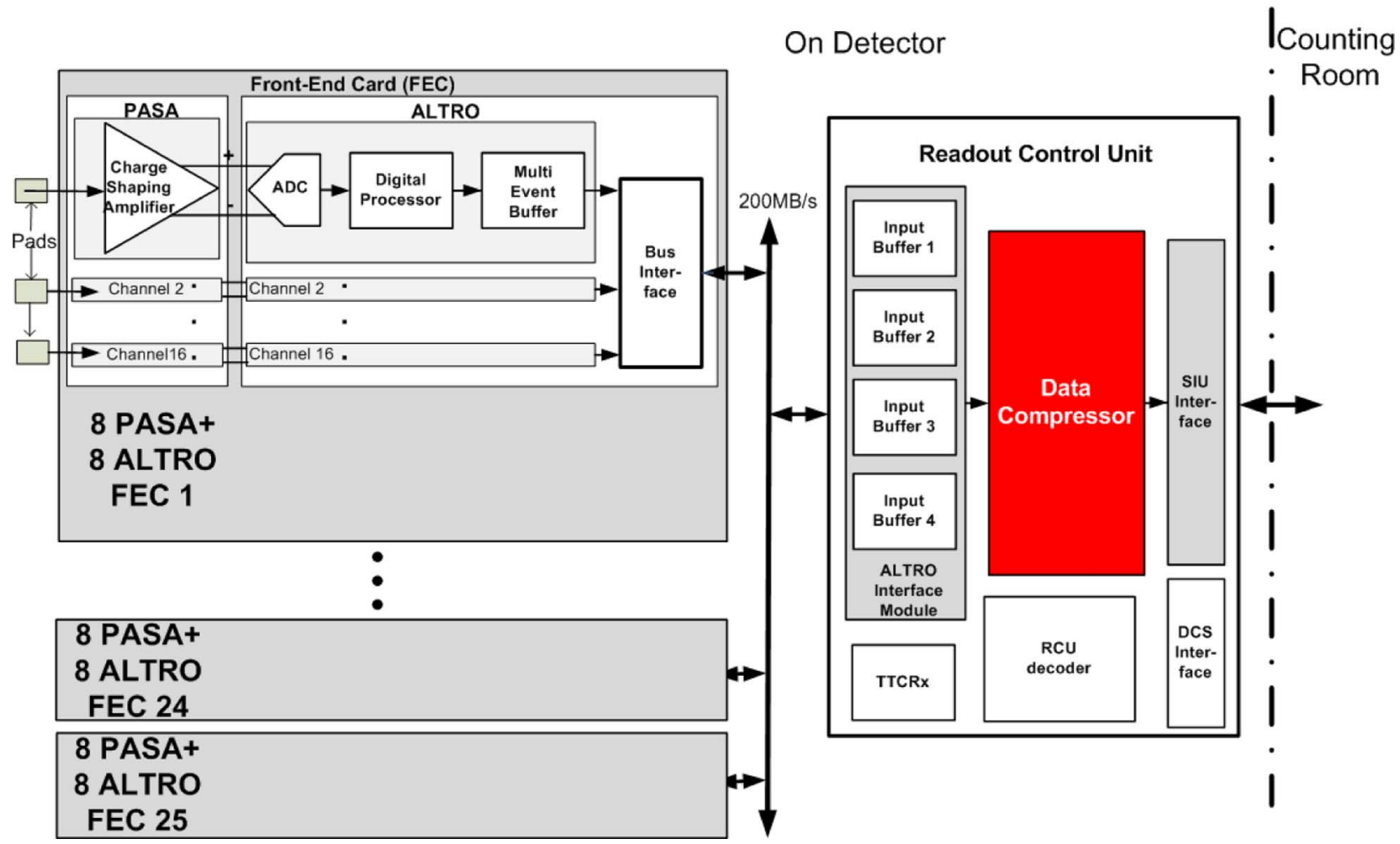

Fig. 1. Simplified block diagram of the ALICE TPC read-out chain showing the location of the proposed compression block.

The compression method developed here is suitable - as an example-for implementation in the Field Programmable Gate Array (FPGA) of the RCU in the TPC front-end electronics [5].

The method is also suitable to be implemented in a future front-end data processing chip such as the ALTRO.

Fig. 1 shows a block diagram illustrating the location where the compression is performed in a typical read-out chain. The ALICE RCU chain is used as a concrete illustration of this. Clearly, the compression operation should be performed prior to sending the data off detector.

\section{THE LOSSLESS COMPRESSION METHOD}

In data compression applications a major distinction can be made between lossy and lossless methods. The zero suppression often used in experiments can cause some loss of information; therefore, to fully preserve the accuracy of the measurement it was decided to focus mainly on lossless compression methods.

After the zero suppression stage performed in the ALTRO, data consist of short vectors of around 10-15 words of 10 bit, representing the sequence of digitized values of the pulses from the channels; each vector is identified with a time stamp. An example of a digitized input pulse is shown in Fig. 2(a).

Normally lossless compression methods use the frequency of occurrence of input symbols to compress them in variable length codes. These methods do not take into account the correlation between symbols and therefore they cannot compress the data better than the limit given by the Shannon entropy [6], [7].

In the case of the TPC data, the sample values are correlated according to the shape of the pulses, which is defined by the analog shaper PASA in the front-end [2]. In order to use this pulse shape and therefore the correlation of the input data, our compression algorithm uses a vector quantization method. The proposed lossless compression algorithm comprises three steps:

- Vector quantization

- Delta calculation

- Huffman coding

Vector quantization method is a generalization of scalar quantization. In normal quantization, an analogue sample, which could in principle have an arbitrary precision, is approximated to a discrete value from a finite set of possible values. In this approximation operation, the discrete value closest to the analog value is usually chosen. In vector quantization instead, a set of continuous analog values (i.e., a vector) is approximated to a set of discrete values by using an appropriate definition of closest approximation [8].

In our case a codebook containing a limited set of reference vectors is created and each input vector is compared to it. The closest approximating reference vector is searched in the codebook and a pointer/index to its position is retained. This method if used alone is considered lossy because the number of reference vectors is limited containing only the most probable input vectors and not all possible input vectors since this would require pointers as large as the input vectors itself creating no compression [6].

Different measures can be used to calculate the distance of an input vector to the reference vectors; the most frequently used is the Euclidian distance. In our case we use the Manhattan distance given in (2), defined as the sum of the absolute values of 


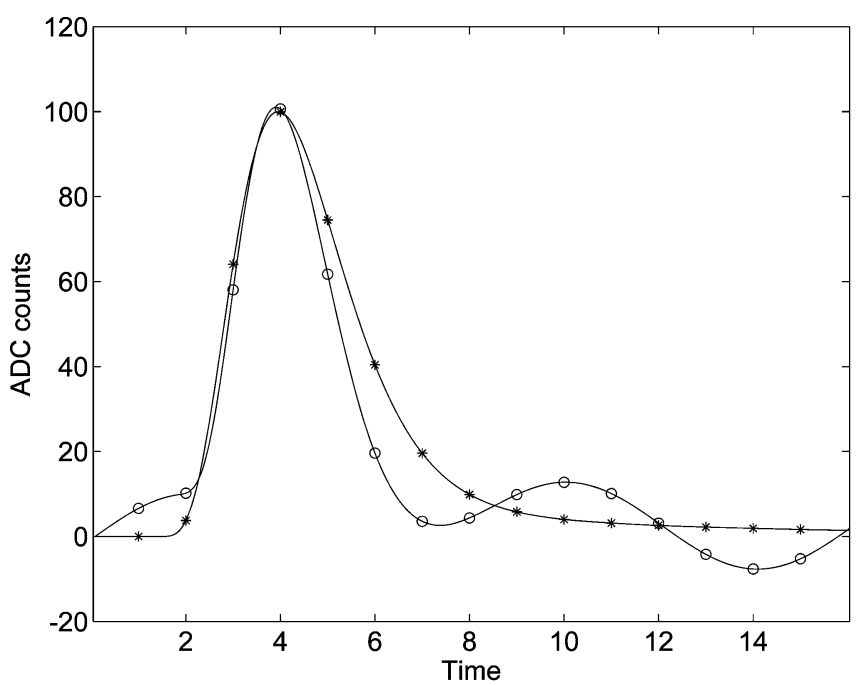

(a)

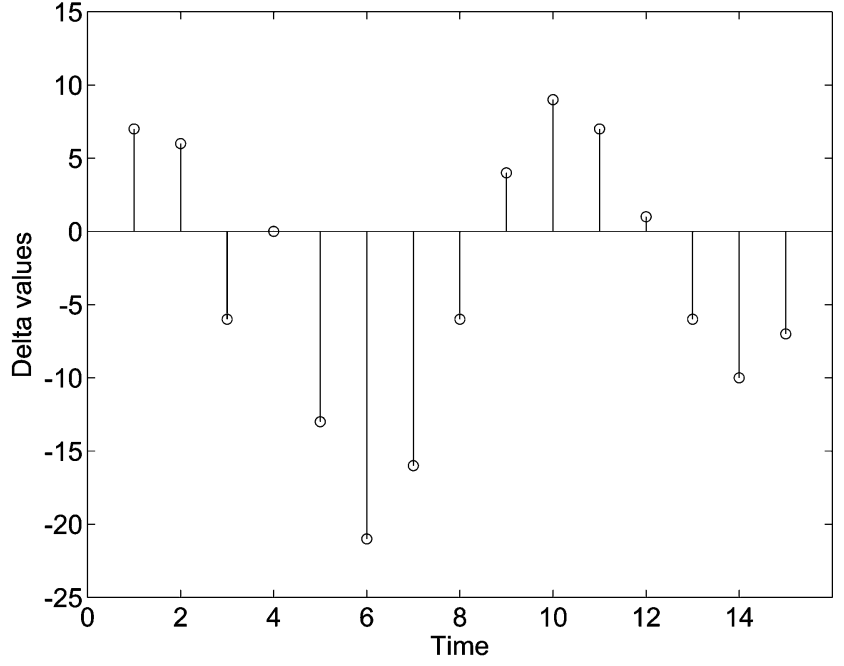

(b)

Fig. 2. Example of a normalized input pulse and a reference pulse and the corresponding delta values (a) Actual input pulse (o) vs. reference pulse $(*)$ (b) Delta values for corresponding example.

the differences between two vectors since this is simpler to implement in hardware [9].

$$
d\left(X, R_{1}\right)=\sum_{i=1}^{n}\left|x_{i}-r 1_{i}\right|
$$

We then find the closest approximating reference vector to the input vector by selecting the

$$
\min \left(d\left(X, R_{1}\right), d\left(X, R_{2}\right), d\left(X, R_{3}\right), d\left(X, R_{4}\right)\right)
$$

$X=\left(x_{1}, x_{2}, \ldots, x_{n}\right)$ is the input vector representing the samples of the pulse. $R_{1} \ldots R_{4}$ are the four reference vectors with the corresponding sample values $r 1_{i}, r 2_{i}, r 3_{i}, r 4_{i}$.

An operation to normalize the input vectors has to be performed prior to the vector quantization, i.e., the amplitude of the peak sample is scaled to a normalized value and all other samples of the vector are subsequently rescaled by the same factor. The normalization factor is included in the output data.

An analysis of the ALICE data sets has shown that four reference vectors are enough to approximate any input data and that no significant further compression could be achieved with more reference vectors. The reference vectors are calculated by segmenting the ALICE data set in four parts, which contain vectors corresponding to input pulses with similar pulse width. The average of the input vectors in each of the four parts is calculated and used as reference vector. The set of the four reference pulses with different widths is shown in Fig. 3.

In order to make the compression lossless, a second operation, the delta calculation is performed where the differences between the samples in the actual input vector and the chosen reference vector are calculated. An example of a reference pulse and a normalized input pulse, as well as the delta values is shown in Fig. 2. The delta values are typically small numbers and can then efficiently be compressed using a lossless entropy

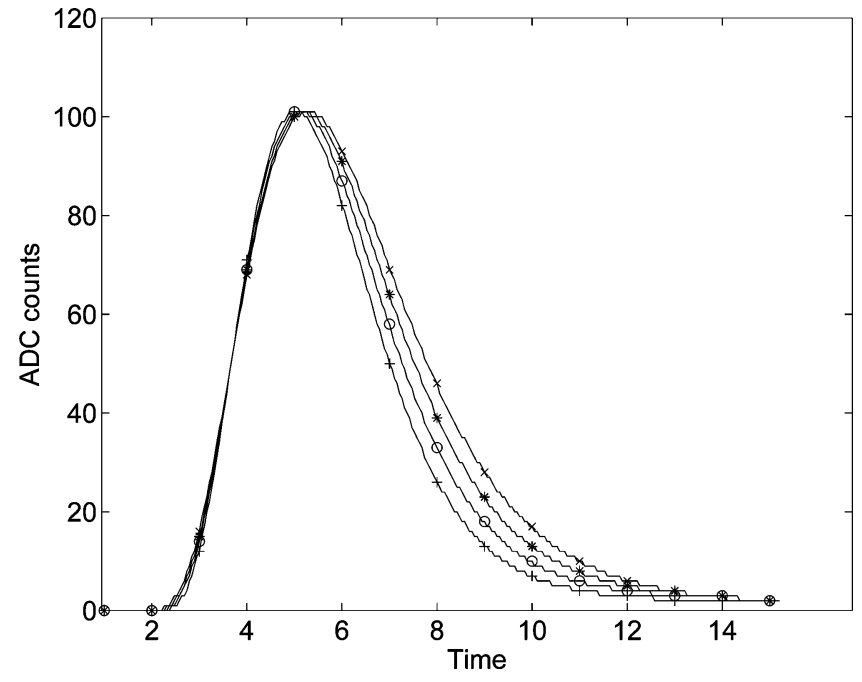

Fig. 3. The four reference pulses.

coding method. For this purpose the Huffman coding algorithm is chosen because of its low complexity.

The Huffman coding uses variable length codewords [6]. Delta values present in the data with higher frequency result in shorter codewords with fewer bits, while delta values with lower frequency are coded in longer codewords. After encoding the whole set of delta values, the average number of bits per symbol can be smaller than by using fixed length codewords. The Huffman coding creates prefix (free) codewords, i.e., no codeword can be a prefix of another codeword [7]. Therefore, in the output bit stream each codeword is uniquely identifiable and can be separated and decoded without using any boundary bits or defined separation codes.

Our implementation of the Huffman algorithm uses a simple fixed mapping (non-adaptive) code where the construction of the delta-data to Huffman codewords mapping is done off-line by using a set of representative data. The resulting Huffman 


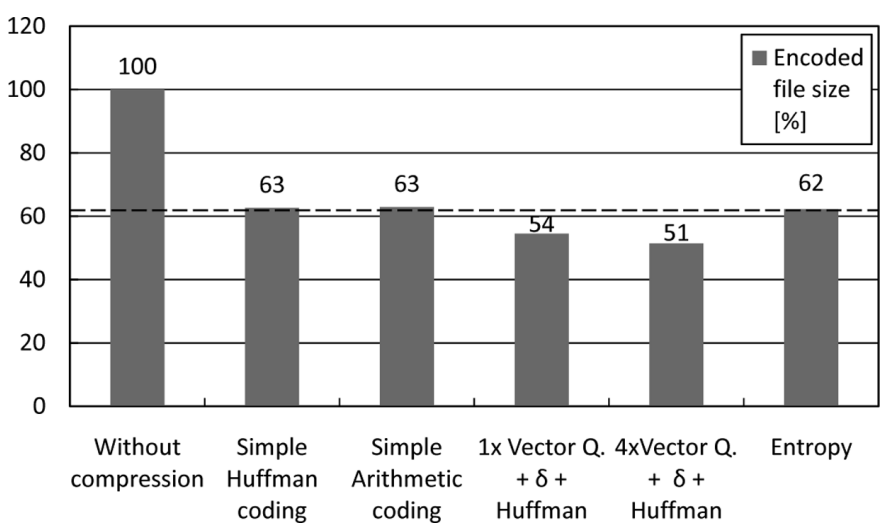

Fig. 4. Efficiency of various lossless compression schemes and computed entropy of the measured data set using Matlab.

codebook is then downloaded to a memory inside the data compression block in an appropriate lookup table.

The performance of our lossless compression algorithm is first investigated by using a Matlab model of the algorithm. This model is used to compress a measured data set from the ALICE TPC obtained by cosmic ray measurements. The data set contains 10,000 pulses and the resulting performance of the compression method is given in the diagram in Fig. 4. For comparison, the diagram also shows the compression efficiencies of other well known lossless compression methods performed on the same data set.

Taking advantage of the vector quantization scheme that uses the correlation of the sample values in a pulse allows us to achieve a compression factor that is better than the simple Shannon entropy for this data set considered as uncorrelated data.

\section{IMPLEMENTATION OF THE ALGORITHM}

A synthesizable model of the Data Compressor block was written in Verilog and has been tested on a development board from Xilinx (ML401) based on a Virtex-4 FPGA [10]. This board offers a wide range of interfaces and a high number of gates and memories inside the FPGA.

The Data Compressor has tree operation modes:

1) Full compression mode (DcMode = "11"): The data is compressed with the proposed algorithm.

2) Huffman Only mode (DcMode = "01"): The data is compressed by using only the Huffman coder.

3) Non-compressing mode (DcMode $=$ "00"): The data is not compressed but bypassed.

A simplified block diagram of the different parts of the Data Compressor is shown in Fig. 5 and is described below.

The Data Compressor module has two clock inputs, one at $40 \mathrm{MHz}$ (Clock40) and one at $80 \mathrm{MHz}$ (Clock80). The $40 \mathrm{MHz}$ clock is the clock used in most parts of the compressor. The input of the Data Compressor consists of 40 bit words coming from the multi-event buffers in the ALTRO chips with a $40 \mathrm{MHz}$ clock. The $80 \mathrm{MHz}$ clock is mainly used for the read-out, to write the compressed data stream into an output FIFO. The normalization operation performed prior to the comparison with the reference vectors requires an integer divider which uses also the $80 \mathrm{MHz}$ clock to reduce latency caused by its pipelined structure.

The sample values of the four reference pulses are stored prior to operation in the RVmem memories inside the Vector Quantizer. The Huffman codebook is stored in two lookup table memories (LUT1, LUT2) and the NormValue and NormValueIndex are saved in the corresponding registers.

The first unit of the compression block is the Normalizer, which will normalize the input vectors coming from the ALTRO chips.

\section{A. Normalizer}

In DcMode = "11" the Normalizer receives up to 4 input samples on each Clock40 cycle. The sample values are saved in a FIFO memory (InputBuffer) while the Normalizer calculates the normalization factor for the input vector. The operation is started by finding the maximum value of each vector. After that, a divider starts dividing the maximum value from the programmed NormValue to calculate the normalization factor $\left(\right.$ NormFactor $=\max \left(\right.$ Vector $\left._{\text {reference }}\right) / \max \left(\right.$ Vector $\left.\left._{\text {input }}\right)\right)$. Afterwards, the NormFactor is scalarly multiplied with the input vector saved in the InputBuffer to normalize its height.

This method requires a 16 bit by 10 bit divider and four 10 bit by 10 bit multipliers to normalize 4 sample values per Clock 40 cycle. The normalization factor NormFactor is rounded to a precision of 10 bit. This limitation in precision of the divider, multipliers and NormFactor introduces a small error in the pulse reconstruction. This error is calculated in Matlab and the results are shown in Section IV.

A formatting unit at the output of the Normalizer is used to align the normalized vectors appropriately with the reference vectors. The Normalizer sends at each Clock 40 cycle up to 4 normalized sample values to the Vector Quantizer.

\section{B. Vector Quantizer}

The four reference vectors in the RVmem memories are first subtracted from the normalized input vector to calculate four sets of delta values.

To find out the best matching reference vector, the resulting four sets are stored in a buffer and the sum of the absolute delta values in each set is calculated. The minimum resulting sum among the four decides which reference vector matched best and the corresponding two-bit index is included in the output data. The corresponding delta values are then sent to the Huffman Coder.

\section{Huffman Coder}

The Huffman Coder receives the input data from two different sources, depending on the mode of operation:

If the Data Compressor works in the full compression mode then the Huffman Coder receives the delta values from the Vector Quantizer.

In case the Huffman Only mode is selected the Huffman Coder receives directly the raw input data. 


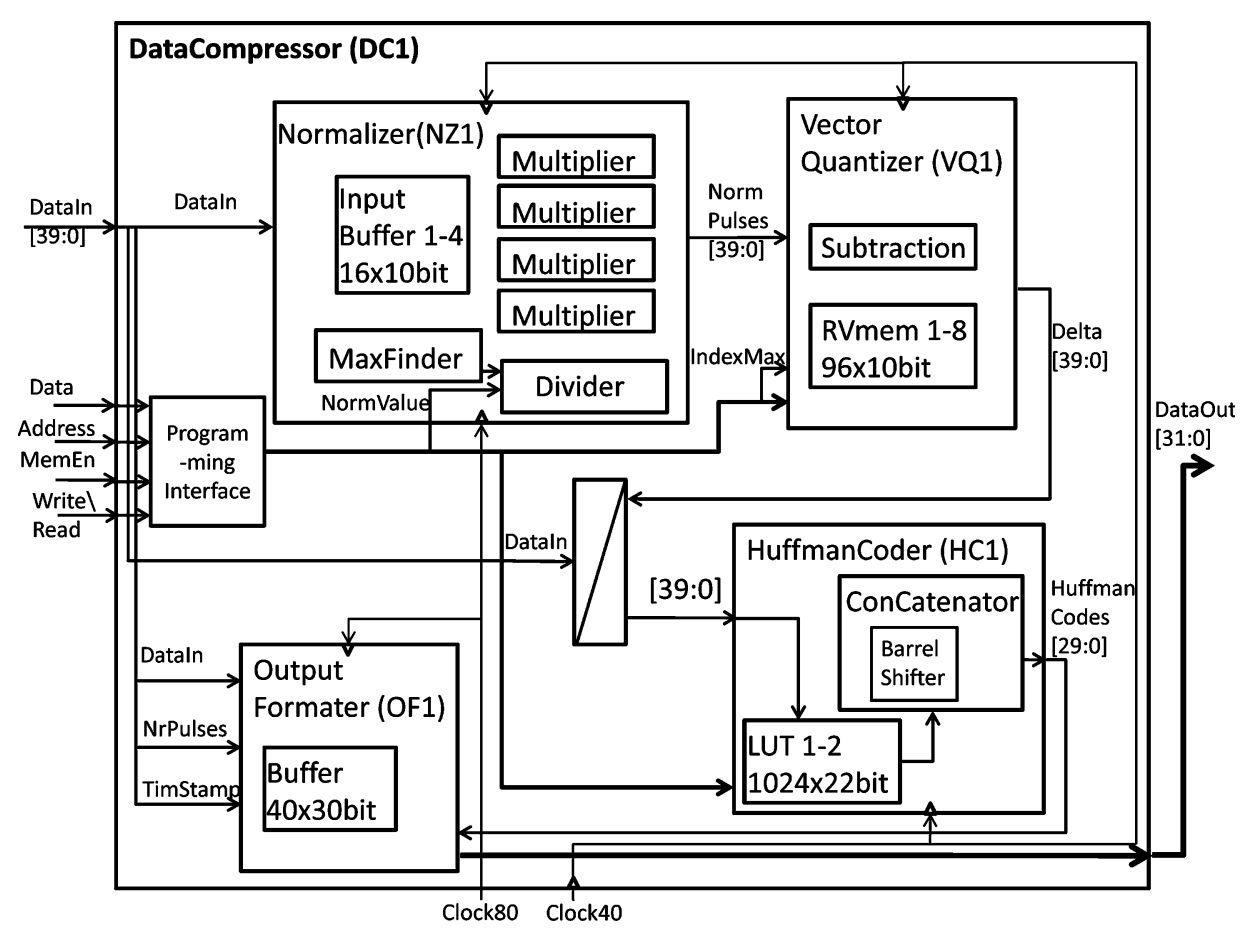

Fig. 5. Simplified block diagram of the data compression implementation.

The Huffman Coder uses the input words to directly address the LUT memories and read out the corresponding Huffman codewords.

Each entry in the LUTs consist of two parts: The five most significant bits are reserved for the Huffman codeword length. The remaining 17 bits are used for the variable length Huffman codewords. The codewords read out from the LUTs are forwarded to the Concatenator module.

The Concatenator joins the variable length Huffman codewords to a continuous output data stream. The output data stream is then saved in blocks of 30 bit wide words into the output FIFO.

\section{Output Formatter}

The final stage of the logic consists of an Output Formatter that prepares the output data block to be sent off detector to the data acquisition system. This block combines the compressed data stream with appropriate headers and flags. The headers contain the number of samples per input vector, the time stamp, the normalization factor and the index of the approximating reference vector. The time stamps preserve the arrival time information of the pulses and the normalization factor is used to rescale the vectors. Before the data is sent off detector, the Output Formatter adds a common data header and a trailer, which contain information about event ID, chip ID, trigger, error codes, etc. [11], [12].

If the Data Compressor works in non-compressing mode, the output data block contains the original data from the ALTRO chips.

\section{RESULTS}

The implementation of the lossless data compression method, presented in this paper, is modeled in Verilog and tested by using a ML401 Xilinx development board [10].

The diagram in Fig. 6 shows the compression efficiency of the implementation according to the different modes of operation. In the full compression mode the compression efficiencies resulting by using either four reference vectors or only one reference vector are compared. In addition, the entropy for the used data set is shown. For a possible future implementation of this compression algorithm in the Virtex-2P FPGA of the RCU in the ALICE TPC only one reference vector can be used to save area. The results are close to the ones achieved with the ideal Matlab model.

The logic that implements our compression scheme has a variable latency essentially generated by the fact that the arrival time of the input signals in a TPC are asynchronous to the system clock and also intrinsically by the fact that Huffman coding produces variable length outputs that cannot always be constrained to the same number of bytes. Still, the implementation of the entire algorithm takes in average 24 clock cycles at $40 \mathrm{MHz}$ (e.g., $0.6 \mu \mathrm{s}$ ) from the first received sample of an input pulse until an output word is sent out. The implementation of the Data Compressor introduces no dead time.

The error caused by the integer precision of the divider and multipliers used in the Normalizer has been evaluated using Matlab. The sample values of the input vectors, which are sent to the compressor block, are fitted in Matlab to form the original input pulses. The same fit-operation is used on the sample values resulting after the compression-decompression operation to obtain the reconstructed pulses. Each original input pulse is 


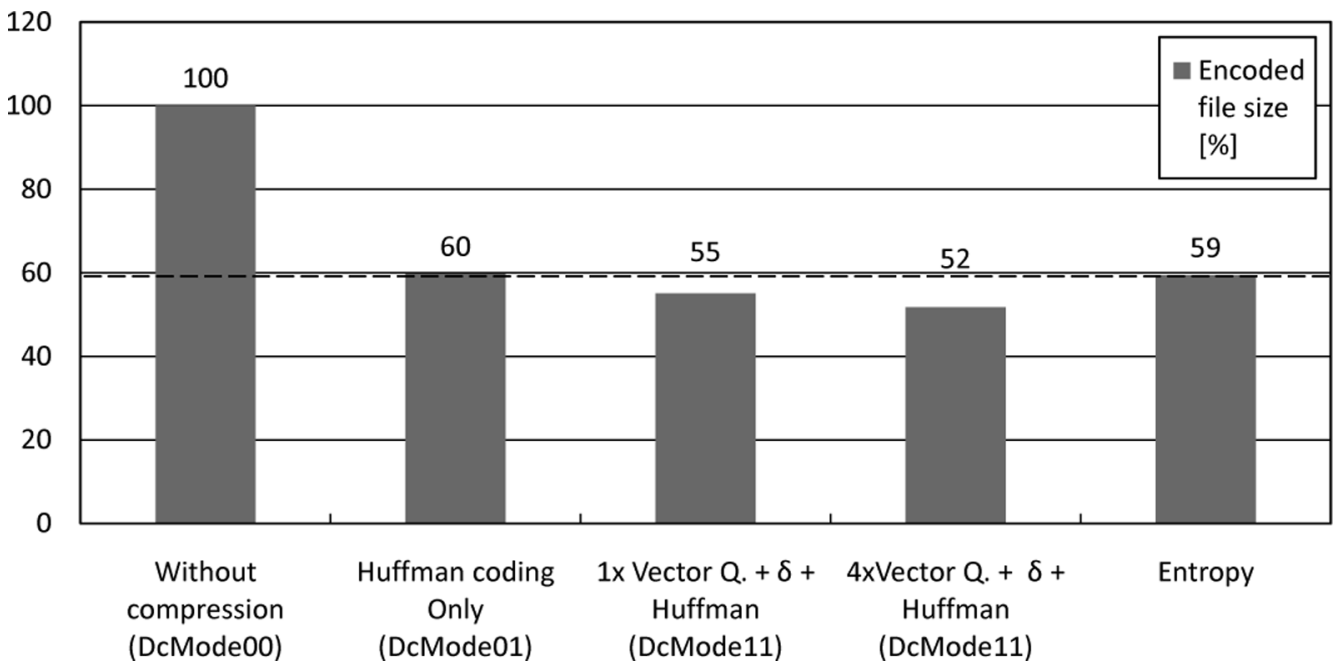

Fig. 6. Compression performance of implementation on zero suppressed data.

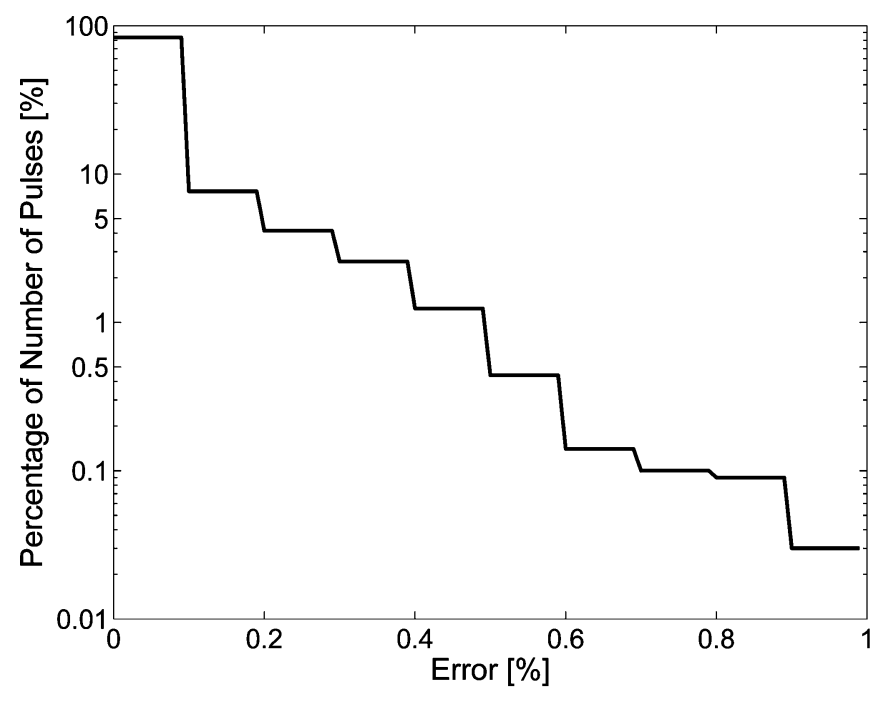

(a)

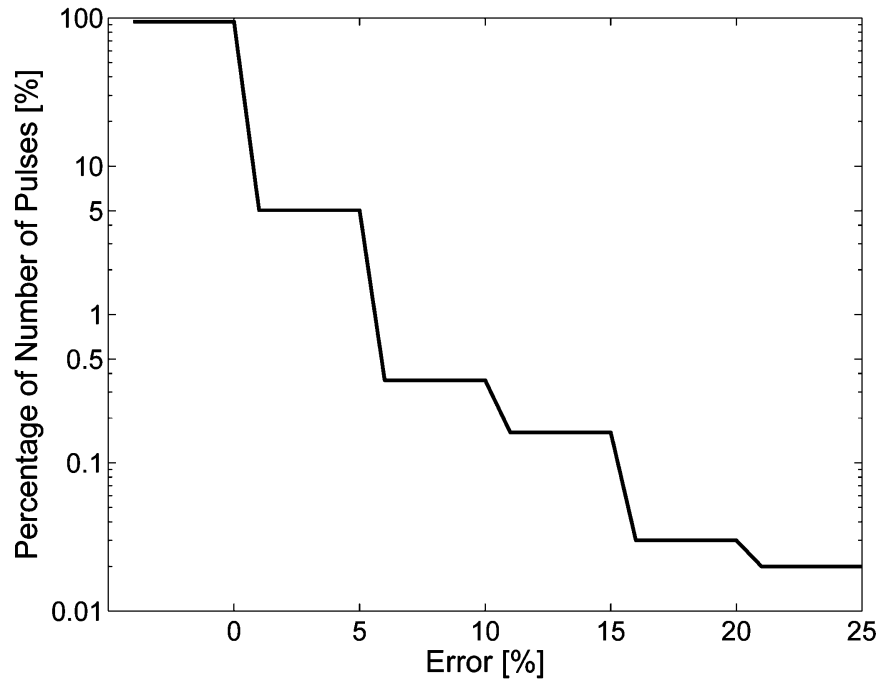

(b)

Fig. 7. Histogram of the error in amplitude (left) and time (right) introduced by the limited precision of the divider, multipliers and NormF actor. The error in amplitude is given in \% of the original amplitude and the error in time is given in nanoseconds. The number of pulses corresponding to the error is given in \% of the total analyzed pulses (a) Amplitude error (b) Time error.

compared to the corresponding reconstructed pulse and the difference in amplitude and time is calculated. The error in amplitude introduced by the Normalizer is defined as the difference expressed in percent to the amplitude of the original input pulse. The error in time results from the difference in position of the maximum expressed in nanoseconds. The error distribution resulting from 10,000 analyzed pulses is shown in the diagram in Fig. 7. Around $85 \%$ of the reconstructed pulses have an error in amplitude less than $0.1 \%$. The remaining pulses have an error above $0.1 \%$, among them only a few pulses have errors up to $1 \%$. No reconstructed pulse was seen with an error in amplitude higher than $1 \%$. The precision in time is even better resulting in around $95 \%$ of the pulses having no time error at the maximum. Around $5 \%$ of the pulses show errors of up to $5 \mathrm{~ns}$. Only a few reconstructed pulses have errors above $5 \mathrm{~ns}$ (up to $25 \mathrm{~ns}$ ). Considering other sources of imprecision in the front-end electronics (ADC, digital signal processor) these errors can be tolerated.
The complexity of the data compression block in terms of resources in an FPGA is summarized below:

$\begin{array}{lr}\text { Number of Slices: } & 2224 \\ \text { Number of Slice Flip Flops: } & 1259 \\ \text { Number of } 4 \text { input LUTs: } & 3958 \\ \text { Number of Block RAMs: } & 10 \\ \text { Number of Multipliers: } & 4\end{array}$

The implementation and optimization of this scheme in a standard cell library for a future inclusion in a new ASIC is currently being prepared.

\section{CONClusions}

A lossless compression method optimized for data from Time Projection Chambers is presented. The scheme uses an application of vector quantization and Huffman coding to digitized data from pulse electronics. 
The results obtained using a Virtex-4 FPGA board proved that the algorithm works as expected and can be implemented in a compact hardware block. This implementation is compatible with the electronics of the ALICE TPC.

The latency of $0.6 \mu \mathrm{s}$ is well suitable for real time applications and the error introduced by the limited precision of the 10-bit integer operations is negligible. This latency can easily be reduced using faster hardware as the method can be further pipelined if necessary.

A data compression factor of $49 \%$ was found. To our knowledge, this compression factor is the highest achieved for lossless methods in High Energy Physics (HEP) applications and allows to reduce significantly the requirements for data transfer bandwidth and storage space in experiments.

\section{REFERENCES}

[1] "The ALICE Time Projection Chamber (TPC)," CERN, 2009. [Online]. Available: http://aliceinfo.cern.ch/Public/en/Chapter2/ Chap2_TPC.html
[2] J. Alme et al., "The ALICE TPC, a large 3-dimensional tracking device with fast readout for ultra-high multiplicity events," Nucl. Instrum. Meth. A, vol. 622, no. 1, pp. 316-367, Jan. 2010.

[3] S. Barras et al., "Time projection chamber ALICE-Technical design report 7," CERN, Geneva, Switzerland, 2000, Tech. Design Rep. LHCC-2000-001.

[4] B. Mota, "Time-domain signal processing algorithms and their implementation in the ALTRO chip for the ALICE TPC," Ph.D. dissertation, ITS, EPFL, Lausanne, Switzerland, 2003.

[5] R. Esteve-Bosch et al., "Readout control unit of the front end electronics for the ALICE time projection chamber," in Proc. 8th Workshop Electron. for LHC Experiments, Colmar, France, 2002, pp. 160-163.

[6] D. Salomon, Data Compression: The Complete Reference, 4th ed. New York: Springer, 2007.

[7] K. Sayood, Introduction to Data Compression, 2nd ed. San Francisco, CA: Morgan Kaufmann, 2000.

[8] A. Gersho and R. M. Gray, Vector Quantization and Signal Compression. Norwell, MA: Kluwer/Springer, 2001.

[9] L. Kandiller, Principles of Mathematics in Operations Research. Ankara, Turkey: Springer, 2006.

[10] "Virtex-4 ML401 evaluation platform," XILINX Inc., 2011. [Online]. Available: http://www.xilinx.com/products/devkits/HW-V4-ML401UNI-G.htm

[11] "RCU Firmware User Manual," V. 2.1, CERN, Geneva, Switzerland, 2010. [Online]. Available: http://ep-ed-alice-tpc.web.cern.ch/ep-edalice-tpc/doc/RCU/DOC/RCUFirmwar2.1.pdf

[12] ALICE TPC Readout Chip User Manual. Geneva, Switzerland: CERN, 2002, Draft 0.2, . 